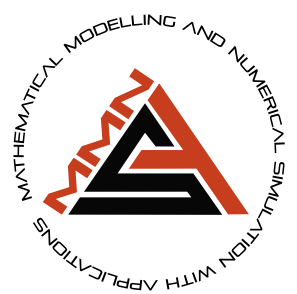

\title{
Vaccination effect conjoint to fraction of avoided contacts for a Sars-Cov-2 mathematical model
}

\author{
Stefania Allegretti $\odot 1,{ }^{\dagger}$, Iulia Martina Bulai $\odot 1,2,{ }^{*},{ }^{\dagger}$, Roberto Marino $\odot 1,{ }^{\dagger}$, \\ Margherita Anna Menandro $1,{ }^{\dagger}$ and Katia Parisi $1,{ }^{\dagger}$
}

${ }^{1}$ Department of Mathematics, Informatics and Economics, University of Basilicata, Viale dell'Ateneo Lucano, 10, 85100 Potenza, Italy, ${ }^{2}$ Member of the research group GNCS of INdAM.

* Corresponding Author

${ }^{\dagger}$ stefania.allegretti@studenti.unibas.it (Stefania Allegretti); iulia.bulai@unibas.it (Iulia Martina Bulai);

roberto.marino@studenti.unibas.it (Roberto Marino); margheritaanna.menandro@studenti.unibas.it (Margherita Anna Menandro); katia.parisi@studenti.unibas.it (Katia Parisi)

\begin{abstract}
In this paper, we consider a modified SIR (susceptible-infected-recovered/removed) model that describes the evolution in time of the infectious disease caused by Sars-Cov-2 (Severe Acute Respiratory Syndrome-Coronavirus-2). We take into consideration that this disease can be both symptomatic and asymptomatic. By formulating a suitable mathematical model via a system of ordinary differential equations (ODEs), we investigate how the vaccination rate and the fraction of avoided contacts affect the population dynamics.
\end{abstract}

Key words: COVID-19; SIR model; asymptomatic cases; avoided contacts; vaccination effect AMS 2020 Classification: 34A34; 92D30; 92D25

\section{Introduction}

The mathematical epidemiology research area, related to modeling infectious diseases, began to develop in 1771 having Daniel Bernoulli as one of the pioneers, [2]. The SIR models and their modified versions are simple tools that can be used to better understand the dynamics of an epidemic, and they gave a significant contribute also for Covid-19 (coronavirus-19 disease) pandemic. The global pandemic status, due to Sars-Cov-2, has been declared, by World Health Organization, at the beginning of 2020, while the virus started to spread around the globe already at the end of 2019 and beginning of 2020 [1].

In the last year an increasing amount of papers for modeling Covid-19 pandemic was published, only to cite few of them see [3]-[31]. The modeling approach helped in a better understanding of the epidemic evolution, such as transmission dynamics of Covid-19 [17]-[19], Covid-19 forecasting, [3], the importance of implementing population-wide interventions, [24]-[25], the role of asymptomatic individuals in the disease transmission, [30]-[31], the vaccination effect on the pandemic outcome, [32]-[34], etc.

Motivated by the importance of a better understanding of the vaccination effect and of the non-pharmaceutical interventions (NPIs) on the disease spreading, here, we consider an extended version of the already studied modified SIR model, [35], considering susceptible individuals, infected individuals that can show symptoms (symptomatic) or not (asymptomatic), and recovered/removed individuals, respectively. The model is characterized by assuming that the infection rate can change depending on NPIs. The novelty here is to consider also the vaccination rate for the susceptible individuals. For a qualitative analysis of the model we compute the equilibrium points and we study their stability by analyzing the Jacobian matrix eigenvalues. We also compute the basic reproduction number. Moreover, for a

> Received: 13.10.2021 > Revised: 18.11.2021 > Accepted: 22.11.2021 > Published: 26.11.2021 
quantitative analysis, via numerical simulation, we investigate how the fraction of avoided contacts and the vaccination rate affects the model outcome, separately using one parameter bifurcation diagrams and jointly by approximating the two strain parameter surface.

The paper outline is as follows. In the first Section, we introduce the model describing all the hypothesis used to build it. In the second Section, we compute a qualitative analysis of the model. In the third Section, via numerical simulations we investigate the importance of both vaccination rate and the fraction of avoided contacts, respectively. Last we present the conclusions of the paper.

\section{Mathematical model formulation}

In this study we introduce a new mathematical model, generalizing the classical SIR model used to describe the transmission and evolution in time of infectious diseases that leads to the immunization of the diseased individual, for the specific case of Sars-Cov-2. In the SIR model we can distinguish three classes of individuals:

Susceptible $H(t)$ : healthy individuals that can get the disease.

Infected $I(t)$ : individuals that are infected and can transmit the disease.

Removed $R(t)$ : individuals that, after being infected, once they recover become immune to the disease, are isolated or died.

For Sars-Cov-2 transmission we consider two different subgroups of the infective classes:

Asymptomatic $A(t)$ : infected individuals that does not present symptoms. We denote with $\phi$ the probability that the disease presents itself in this form.

Symptomatic $S(t)$ : infected individuals that present symptoms. The probability that the disease manifests itself in this form is $1-\phi$.

From now on for simplicity we will abbreviate the new model with SASR (Susceptible-Asymptomatic-Symptomatic-Removed). We assume to have a constant total population in time, $N$, this is reasonable for two reasons: (i) if we consider the beginning of the epidemic it means that only a short interval of time will be considered; (ii) while if we consider a long time after the onset of the epidemic we can assume that the mortality rate due to the disease is lower and lower due to a better understanding of the virus and improvement of the effects of the cure. We also consider the demographic parameters such as constant birth/immigration term, $\Omega$, in the susceptible class and a mortality rate, $\mu_{N}$, due to other causes besides the disease, present in all the considered classes.

The infection rate take into consideration also the effect of non-pharmaceutical interventions (NPIs) by means of a parameter $\psi$, the fraction of avoided contacts, the infection rate reads $\beta=\lambda(1-\psi)$. Here we assume that the contact rate between susceptible and infected is reduced thanks to NPIs adopted by individuals or by institutions, in order to avoid the contagion.

Once infected, a fraction $1-\phi$ of individuals can develop symptoms and the remaining ones $\phi$ stay asymptomatic. We also assume that the asymptomatic individuals can develop symptoms at rate $\delta$. Last, we assume that both asymptomatic and symptomatic individuals can move in the removed class at rate $\gamma_{A}$ and $\gamma_{S}$, respectively, and that exist a vaccine and the susceptible individuals can be vaccinated and get a permanent immunity at rate $\mu$.

Given the assumptions introduced above the model reads:

$$
\begin{aligned}
& \frac{d H}{d t}=\Omega-\beta \frac{H(A+S)}{N}-\mu_{N} H-\mu H, \\
& \frac{d A}{d t}=\phi \beta \frac{H(A+S)}{N}-\gamma_{A} A-\mu_{N} A-\delta A, \\
& \frac{d S}{d t}=(1-\phi) \beta \frac{H(A+S)}{N}-\gamma_{S} S-\mu_{N} S+\delta A-\mu_{S} S, \\
& \frac{d R}{d t}=\gamma_{A} A+\gamma_{S} S-\mu_{N} R+\mu H,
\end{aligned}
$$

with $\phi, \psi \in[0,1]$. In Figure 1, we have represented a sketch of the main interactions between the four classes of the SASR model.

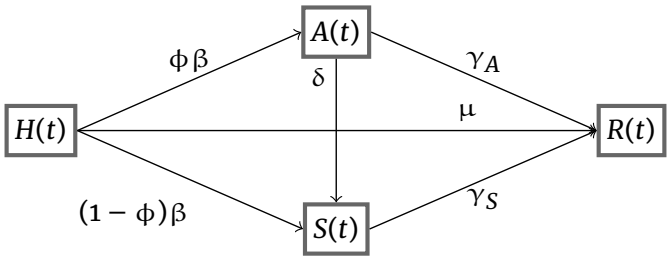

Figure 1. The diagram for the main interaction between the four classes of the SASR model, without considering the mortality rates and birth/immigration term.

\section{Qualitative analysis of the model}

\section{Boundedness}

It is important to establish that the variables cannot grow unbounded. We show now that the system's trajectories remain within a compact set. We consider the function

$$
\varphi(t)=H(t)+A(t)+S(t)+R(t) .
$$


Summing up the equations in (1), we then have

$$
\frac{d \varphi(t)}{d t}+\mu_{N} \varphi(t)=\Omega-\mu_{S} S \quad \Leftrightarrow \quad \frac{d \varphi(t)}{d t}+\mu_{N} \varphi(t) \leq \Omega .
$$

Since $\varphi(0)=N$, we can solve the corresponding differential equation, and find that:

$$
\varphi(t) \leq \max \left\{\frac{\Omega}{\mu_{N}}, N\right\}
$$

which guarantees that every single variable must have the same upper bound as well.

Equilibrium points

In order to find the equilibrium points we assume $a=\gamma_{A}+\mu_{N}+\delta, b=\gamma_{S}+\mu_{S}+\mu_{N}$ and $W=\frac{H(A+S)}{N}$ in (1), and get the new simplified version of the model by equating to zero the right hand side of the obtained model:

$$
\left\{\begin{array}{l}
\Omega-\beta W-\mu_{N} H-\mu H=0 \\
\phi \beta W-a A=0 \\
(1-\phi) \beta W-b S+\delta A=0 \\
\gamma_{A} A+\gamma_{S} S-\mu_{N} R+\mu H=0 \\
W=\frac{H(A+S)}{N}
\end{array}\right.
$$

which is equivalent to

$$
\left\{\begin{array}{l}
H=\frac{\Omega-\beta W}{\mu_{N}+\mu} \\
A=\frac{\phi \beta W}{a}, \\
S=\frac{\beta W(a(1-\phi)+\delta \phi)}{a b}, \\
\gamma_{A}\left(\frac{\phi \beta W}{a}\right)+\gamma_{S}\left(\frac{\beta W(a(1-\phi)+\delta \phi)}{a b}\right)-\mu_{N} R+\mu\left(\frac{\Omega-\beta W}{\mu_{N}+\mu}\right)=0 \\
W=\frac{\left(\frac{\Omega-\beta W}{\mu_{N}+\mu}\right)\left(\frac{\phi \beta W}{a}+\frac{\beta W(a(1-\phi)+\delta \phi)}{a b}\right)}{N}
\end{array}\right.
$$

Solving the last equation of (3) we get

$$
\begin{aligned}
& W_{1}=0 \\
& \text { or } \\
& W_{2}=\frac{-\phi \beta b \Omega-a \beta \Omega+a \beta \phi \Omega-\delta \phi \beta \Omega+N a b\left(\mu_{N}+\mu\right)}{\beta^{2}(-\phi b-a+a \phi-\delta \phi)} .
\end{aligned}
$$

- For $W_{1}$ we get the disease free equilibrium (DFE)

$$
E_{0}=\left(H_{0}, A_{0}, S_{0}, R_{0}\right)=\left(\frac{\Omega}{\mu_{N}+\mu}, 0,0, \frac{\mu \Omega}{\mu_{N}\left(\mu_{N}+\mu\right)}\right),
$$

that is always feasible.

- For $W_{2}$ we get the coexistence equilibrium

$$
E_{*}=\left(H_{*}, A_{*}, S_{*}, R_{*}\right)
$$

with

$$
\begin{aligned}
H_{*} & =\frac{N a b}{\beta(a(1-\phi)+\phi(b+\delta))}, \\
A_{*} & =\frac{\phi\left[\beta \Omega(a(1-\phi)+\phi(b+\delta))-N a b\left(\mu_{N}+\mu\right)\right]}{a \beta(a(1-\phi)+\phi(b+\delta))}, \\
S_{*} & =\frac{(a(1-\phi)+\delta \phi)\left[\beta \Omega(a(1-\phi)+\phi(b+\delta))-N a b\left(\mu_{N}+\mu\right)\right]}{a b \beta(a(1-\phi)+\phi(b+\delta))}, \\
R_{*} & =\frac{\left(b \gamma_{A} \phi+\gamma_{S}(a(1-\phi)+\delta \phi)\left[\Omega \beta(a(1-\phi)+\phi(b+\delta))-N a b\left(\mu_{N}+\mu\right)\right]-\mu N a^{2} b^{2}\right.}{\mu_{N} a b \beta(a(1-\phi)+\phi(b+\delta))} .
\end{aligned}
$$

Notice that $H_{*}>0$, while 


$$
A_{*}>0 \quad \Leftrightarrow \quad \frac{\phi\left[\beta \Omega(a(1-\phi)+\phi(b+\delta))-N a b\left(\mu_{N}+\mu\right)\right]}{a \beta(a(1-\phi)+\phi(b+\delta))}>0,
$$

solving the inequality for $\mu$ we get that

$$
\mu<\frac{\beta \Omega(a(1-\phi)+\phi(b+\delta))}{N a b}-\mu_{N}
$$

must hold. Assuming $A_{*}>0$ also $S_{*}>0$ and $R_{*}>0$ hold and the coexistence equilibrium $E_{*}$ is feasible.

Jacobian matrix and characteristic polynomial

In order to study the stability of the equilibrium points we need to compute the eigenvalues of the Jacobian matrix associated to system (1) evaluated at the equilibrium points. The Jacobian matrix is

$$
J=\left(\begin{array}{cccc}
-\beta \frac{(A+S)}{N}-\mu_{N}-\mu & -\beta \frac{H}{N} & -\beta \frac{H}{N} & 0 \\
\phi \beta \frac{(A+S)}{N} & \phi \beta \frac{H}{N}-a & \phi \beta \frac{H}{N} & 0 \\
(1-\phi) \beta \frac{(A+S)}{N} & (1-\phi) \beta \frac{H}{N}+\delta & (1-\phi) \beta \frac{H}{N}-b & 0 \\
\mu & \gamma_{A} & \gamma_{S} & -\mu_{N}
\end{array}\right) .
$$

We compute the characteristic polynomial associated to $J$ by computing $\operatorname{det}(J-\chi I)$, and we get

$$
\begin{aligned}
p(x)=\frac{\left(-\mu_{N}-x\right)}{N} \cdot\left[N x^{3}+\chi^{2}\left(\beta(A+S-H)+N\left(a+b+\mu_{N}+\mu\right)\right)+\right. & \\
+\chi\left(-\beta H\left(\mu_{N}+\mu+a(1-\phi)+\phi(b+\delta)\right)+\beta(A+S)(a+b)+N\left(a b+\left(\mu_{N}+\mu\right)(a+b)\right)\right)+ & \left.-\beta H\left(\left(\mu_{N}+\mu\right)(a(1-\phi)+\phi(b+\delta))\right)+a b \beta(A+S)+N a b\left(\mu_{N}+\mu\right)\right] .
\end{aligned}
$$

Substituting in (5) the values of $E_{0}$ we get

$$
p_{0}(x)=\left(\mu_{N}+x\right)\left(\mu_{N}+\mu+x\right)\left[x^{2}+\left(a+b-\frac{\beta \Omega}{N\left(\mu_{N}+\mu\right)}\right) x+a b-\frac{\beta \Omega[a(1-\phi)+\phi(b+\delta)]}{N\left(\mu_{N}+\mu\right)}\right]
$$

that has two negative eigenvalues $x_{1}=-\mu_{N}$ e $x_{2}=-\left(\mu_{N}+\mu\right)$. In order to have a stable DFE we should analyze the sign of the real parts of the roots of the second degree polynomial

$$
x^{2}+\left(a+b-\frac{\beta \Omega}{N\left(\mu_{N}+\mu\right)}\right) x+a b-\frac{\beta \Omega[a(1-\phi)+\phi(b+\delta)]}{N\left(\mu_{N}+\mu\right)} .
$$

Notice that, $\forall \phi, \psi \in[0,1]$, the two roots of (6) are real. Imposing the second and the third coefficients of (6) to be positive and solving with respect to the vaccination rate, $\mu$, we get the condition

$$
\mu>\max \left\{\frac{\beta \Omega}{N(a+b)}-\mu_{N}, \quad \frac{\beta \Omega[a(1-\phi)+\phi(b+\delta)]}{N a b}-\mu_{N}\right\}
$$

that guaranties that the second degree equation, (6), has two negative real roots and thus the stability of $E_{0}$. In analogues way we study the stability of the coexistence equilibrium. We evaluate the Jacobian matrix (4) at $E_{*}$ and we compute the associated characteristic polynomial

$$
p_{*_{\mu}}(x)=\left(\mu_{N}+x\right)\left(x^{3}+a_{2} x^{2}+a_{1} x+a_{0}\right)
$$

with

$$
\begin{aligned}
& a_{2}=\frac{1}{N}\left[\frac{N a b}{(a(1-\phi)+\phi(b+\delta))}+\frac{\beta \Omega(a(1-\phi)+\phi(b+\delta))}{a b}+N(a+b)\right], \\
& a_{1}=\frac{1}{N}\left[\frac{-N a b\left(\mu_{N}+\mu\right)}{(a(1-\phi)+\phi(b+\delta))}+\frac{[\beta \Omega(a(1-\phi)+\phi(b+\delta))](a+b)}{a b}\right], \\
& a_{0}=\frac{1}{N}\left[\beta \Omega(a(1-\phi)+\phi(b+\delta))-N a b\left(\mu_{N}+\mu\right)\right] .
\end{aligned}
$$

The root $x_{1}=-\mu_{N}$ is always negative while for the coexistence equilibrium to be stable the Routh-Hurwitz criterion must hold $a_{0}>0$ (true if the equilibrium is feasible), $a_{2}>0$ (true) and $a_{1} a_{2}>a_{0}$. 
Table 1. Parameters of the model for data considering Italy. ${ }^{a}[36]\left(\right.$ ISTAT 2018), ${ }^{b} \Omega$ was chosen such that $H(0) \simeq \Omega / \mu_{N},{ }^{c}[38],{ }^{d}[39],{ }^{e}$ Fitted using data from [37].

\begin{tabular}{crrr}
\hline Parameters & Name & Value & Unit \\
\hline$N$ & total population & $60.36 \times 10^{6 a}$ & human \\
$\Omega$ & birth and immigration & $633780^{b}$ & human/day \\
$\lambda$ & infection rate & $0.292^{c}$ & day $^{-1}$ \\
$\psi$ & test & pure number \\
$\phi$ & prob. of undergoing asympt. infection & $0.5^{d}$ & pure number \\
$\gamma_{A}$ & per capita recovery rate A & 0.028 & day $^{-1}$ \\
$\gamma_{S}$ & per capita recovery rate S & $0.028^{e}$ & day $^{-1}$ \\
$\mu_{N}$ & mortality rate due to other causes & $0.0105^{a}$ & day $^{-1}$ \\
$\mu$ & vaccination rate & test & day $^{-1}$ \\
$\delta$ & transition from $A \rightarrow S$ & $0.067^{d}$ & day $^{-1}$ \\
$\mu_{S}$ & mortality rate due Covid-19 & $0.0069^{e}$ & day $^{-1}$ \\
$a$ & $\gamma_{A}+\mu_{N}+\delta$ & 0.1055 & day $^{-1}$ \\
$b$ & $\gamma_{S}+\mu_{S}+\mu_{N}$ & 0.0454 & day $^{-1}$ \\
\hline
\end{tabular}

\section{Basic reproduction number $R_{0}$}

The basic reproduction number, $R_{0}$, is "the expected number of secondary cases produced, in a completely susceptible population, by a typical infective individual", (e.g. [40]). The importance of $R_{0}$ in the spreading of a disease is related to its value. The ideal scenario is $R_{0}<1$, in this case the infection cannot grow. This means that on average an infected individual produces less than one new infected individual over the course of its infectious period. Conversely if $R_{0}>1$, the disease spread over the population, in fact each infected individual produces, on average, more than one new infection. We compute the basic reproduction number using the next generation matrix technique, (for a detailed description of the method see [40], [41]), and we get

$$
R_{0}=\frac{\lambda(1-\psi) \Omega}{\left(\mu_{N}+\mu\right) N a b}[(1-\phi) a+\phi(b+\delta)]
$$

where we used that $\beta=\lambda(1-\psi)$. From (8) one can see that also in presence of the vaccine the epidemic can evolve and the stability of the coexistence equilibrium is reached. In order to have the stability of the DFE the vaccination efficiency must be greater than a certain threshold

$$
\mu>\left(R_{0}-1\right) \mu_{N}=\frac{\lambda(1-\psi) \Omega(a(1-\phi)+\phi(b+\delta))}{N a b}-\mu_{N}
$$

For values of $\mu$ for which (9) does not hold the disease spread and the coexistence equilibrium stability is reached.

\section{Numerical analysis of the model}

In this section we will analyze, from a numerical perspective, how the vaccination rate and the fraction of avoided contacts affects the solutions of the system of ordinary differential equations, defined in (1). We also find the transcritical bifurcation value for $\mu$ fixing all the other parameter values as in Table 1 and $\psi=0$. Assuming that $\mu=0$, no vaccination is available, we investigate the importance of the fraction of avoided contact parameter, $\psi$. In Figure 2 are reported the solutions of system (1) for 5 different values of $\psi$ in $[0,1]$ with step 0.2. Notice that if $\psi=1$, meaning that the virus does not circulate and the infection rate is zero, the DFE become stable, on the other side for $\psi=0$ no measures to avoid contact are taken and the coexistence equilibrium reach its stability. It is worth noting that increasing the NPIs the maximum value of the peak in the asymptomatic and symptomatic populations not only decrease but is also shifted to the right, so there is a delay which can give an advantage in those situations where the ICU (Intensive Care Units) are overloads. In Figure 3 we have plotted the six numerical solutions of the ODE system (1), fixing all the parameter values as in Table $1, \psi=0$ and $\mu$ assuming 6 different values in the interval $[0,0.5]$ with step 0.1. Notice that without a vaccine $(\mu=0)$ both asymptomatic and symptomatic individuals reaches their highest peak, with all the other solution pressed against the abscissa axis, though they are not zero. In fact in Figure 4 we have reported a zoomed version of this two populations for values of $\mu$ much closer to 0 , that confirm the stability of the coexistence equilibrium (for the first three lowest values) where the disease it is not yet eradicated and for $\mu=0.06$ the stability of the DFE. In Figure 5 we have plotted one parameter bifurcation diagram with respect to $\mu$ (left panel) and $\psi$ (right panel), respectively. For $\mu \simeq 0.059$ (or for $\psi \simeq 0.81$ ) a transcritical bifurcation arises and for system (1) the coexistence equilibrium interchanges its stability with the disease free equilibrium. In Figure 6 we have represented a two strain parameter plot with respect to both $\mu$ and $\psi$. We can see that without vaccination the system reach the DFE stability only for values of the fraction of avoided contact close to 1 , that means strict measures are needed in order to have an infection rate close to 0 . Moreover if we assume that the fraction of avoided contacts is 0 , which means no measures are taken, the DFE it is stable for a vaccination rate higher than 0.06 ( $\leqslant 17$ days). In Figure 7 we represented the contour plots of the surfaces introduced in Figure 6. 

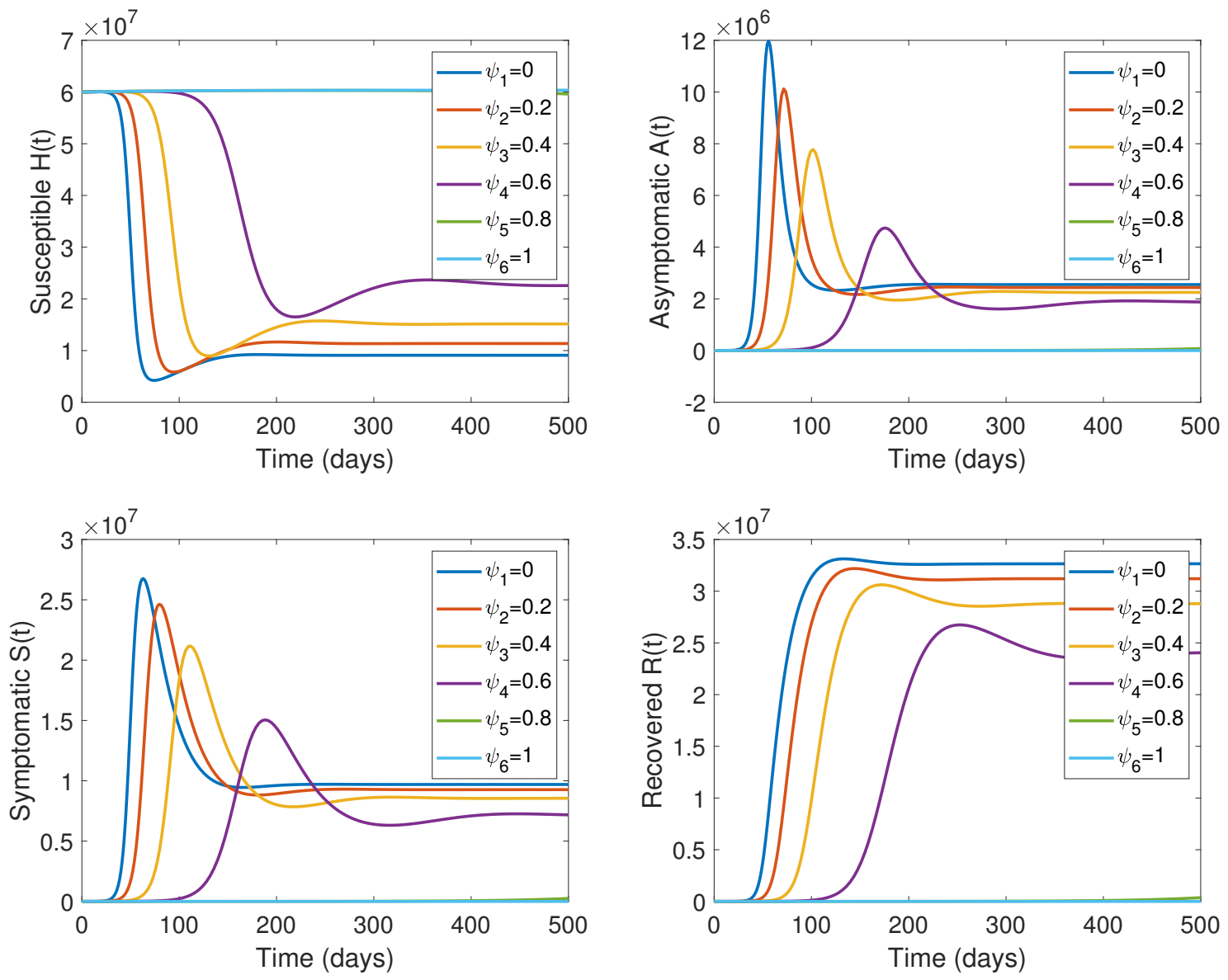

Figure 2. The numerical solutions of system (1) fixing all the parameter values as in Table $1, \mu=0$ (no vaccination) and $\psi$ assuming 6 different values in the interval [0, 1$]$ with step 0.2. Top row: Susceptible individuals in time (left panel) and asymptomatic individuals in time (right panel). Bottom row: symptomatic individuals in time (left panel) and recovered/removed individuals in time (right panel).

\section{Conclusions}

In this paper we have introduced a SASR (Susceptible-Asymptomatic-Symptomatic-Recovered/Removed) model to describe the dynamics of four different classes of individuals where Sars-Cov-2 virus infection is considered. In this model we have also considered the vaccination rate and a parameter in the infection rate that represent the avoided contacts between individuals due to NPIs. We computed the disease free equilibrium and the coexistence equilibrium and analyzed their local stability. Moreover we have computed the basic reproduction number.

From the numerical investigation we can conclude that: (i) increasing the fraction of avoided contacts $\psi$ leads to, not only to delay the peak, but also to lower the maximum value, with a direct consequence on decreasing the pressure on the ICU; (ii) assuming to have an efficient vaccine with a permanent immunity, we found a critical value for the vaccination rate, bellow which the disease free equilibrium is locally asymptotically stable, while if above this threshold we have the confirmation that higher the efficiency of vaccine lower the peak of infected individuals at the coexistence equilibrium. From the two strain parameter analysis we can conclude that both an efficient vaccine and a high fraction of avoided contacts lead to the stability of the disease free equilibrium, but also that higher the efficiency of the vaccine smaller the fraction of avoided contact must be. 

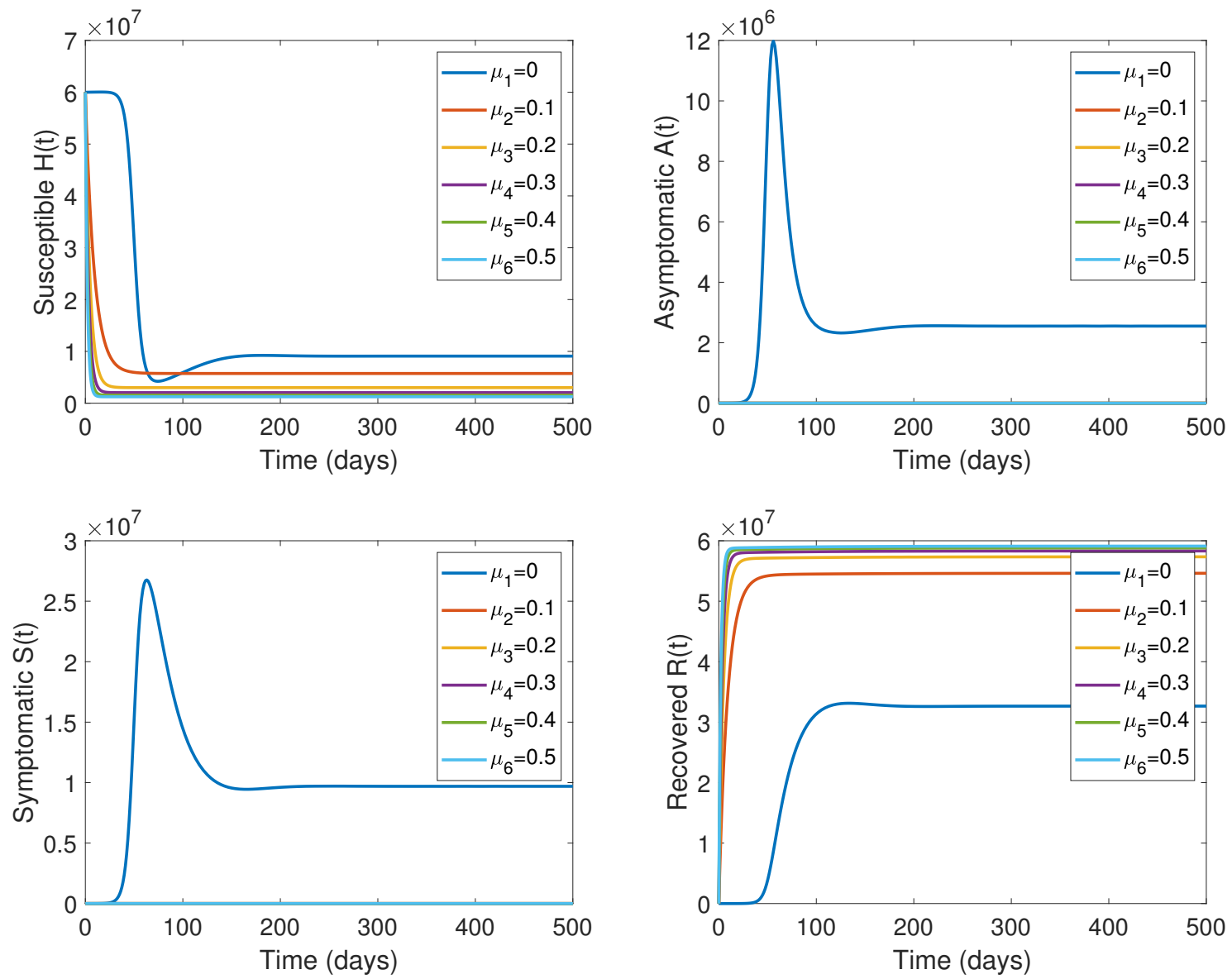

Figure 3. The numerical solutions of system (1) fixing all the parameter values as in Table $1, \psi=0$ and $\mu$ assuming 6 different values in the interval [0, 0.5] with step 0.1. Top row: Susceptible individuals in time (left panel) and asymptomatic individuals in time (right panel). Bottom row: symptomatic individuals in time (left panel) and recovered/removed individuals in time (right panel).
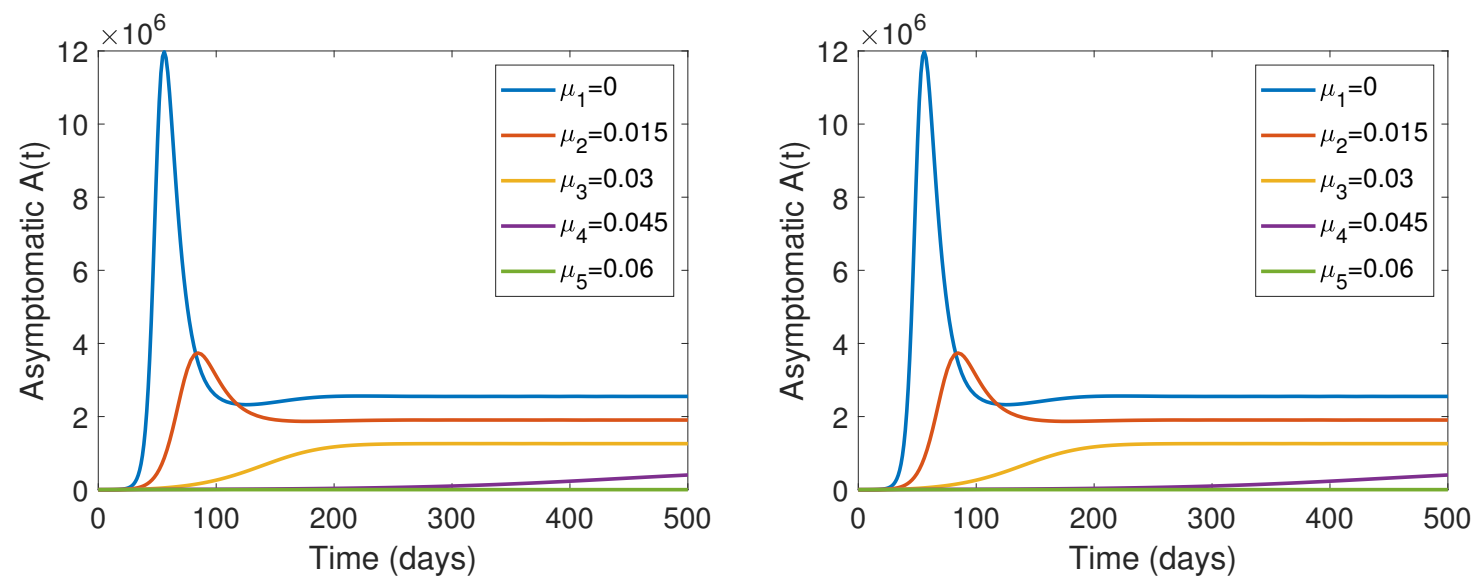

Figure 4. Zoomed version of Figure 3 for asymptomatic (left) and symptomatic (right) populations, respectively, assuming $\mu$ varying in [0, 0.06] with step 0.015 .

\section{Declarations}

Availability of data and material

Not applicable.

Consent for publication

Not applicable. 

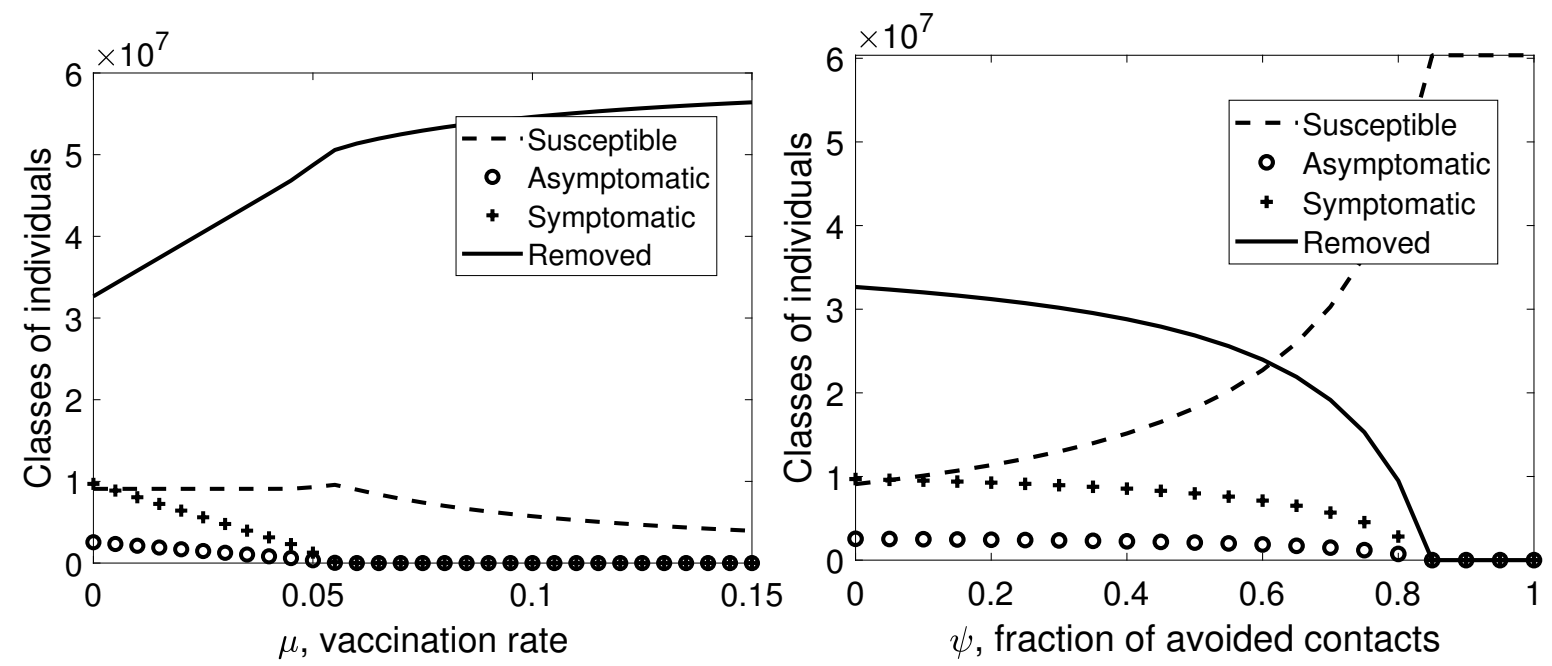

Figure 5. One parameter bifurcation diagram with respect to the vaccination rate $\mu$ (left panel) and to fraction of avoided contacts $\psi$ (right panel). All the other parameter values fixed as in Table 1, $\psi=0$ (left panel) and $\mu=0$ (right panel). For $\mu \simeq 0.059$ (and $\psi \simeq 0.81$ ) a transcritical bifurcation arises, (the coexistence equilibrium interchanges its stability with the disease free equilibrium).
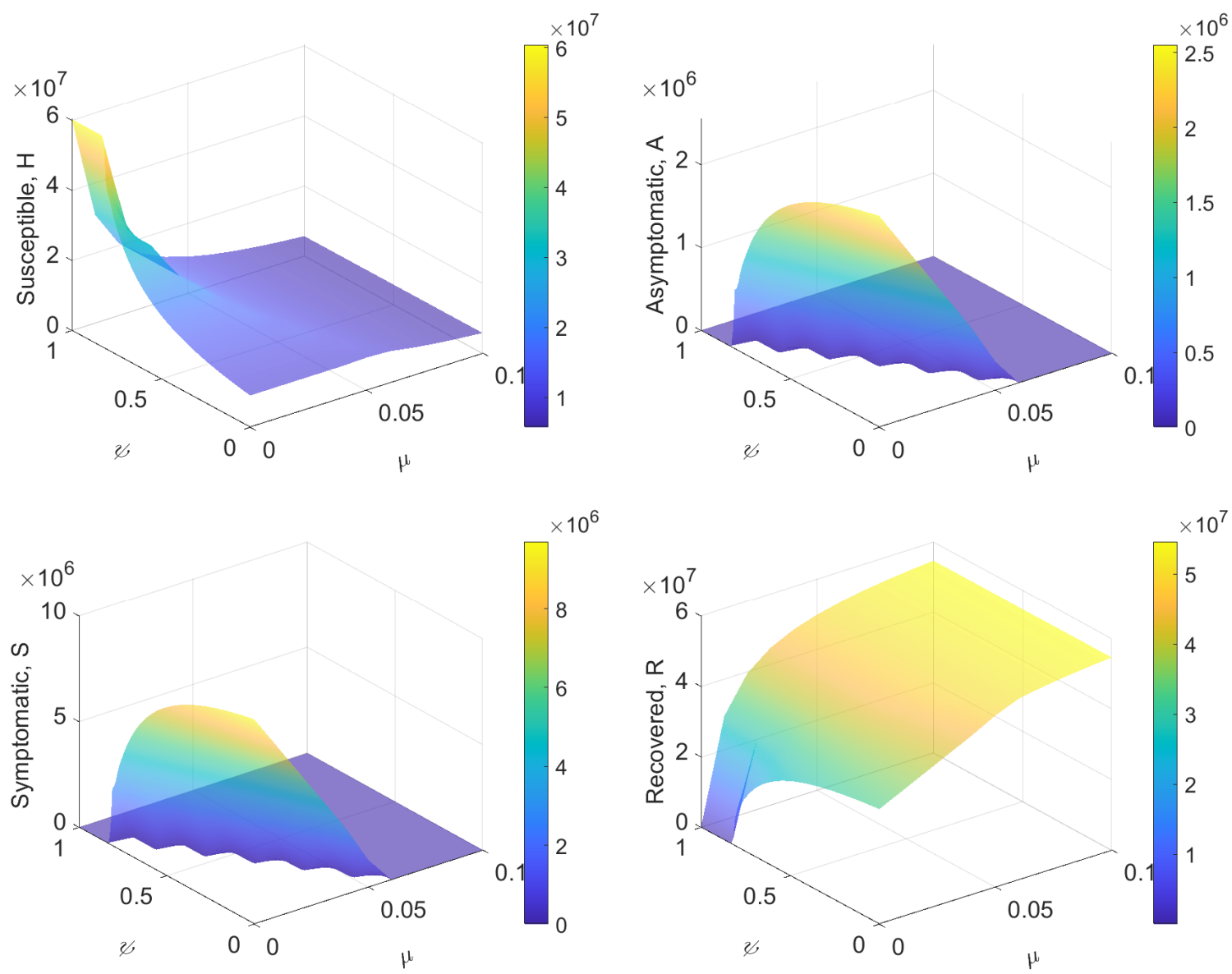

Figure 6. The numerical solutions of system (1) fixing all the parameter values as in Table 1 , and varying $\psi \in[0,1]$ and $\mu \in[0,0.1]$. Top row: Susceptible individuals (left panel) and Asymptomatic individuals (right panel). Bottom row: Symptomatic individuals (left panel) and Recovered individuals (right panel).

Conflicts of interest

The authors declare that they have no conflict of interests. 

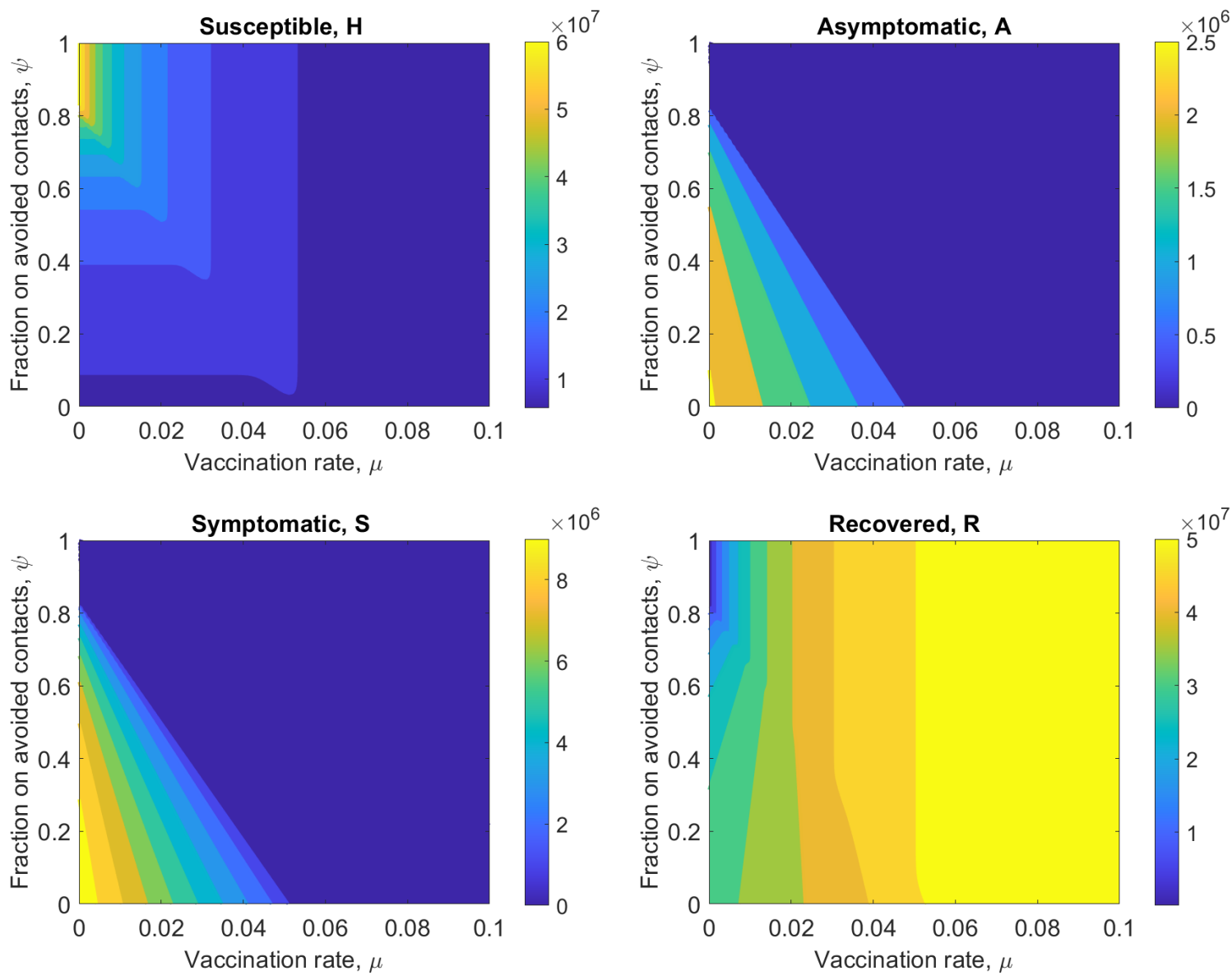

Figure 7. Contour plot of the surfaces represented in Figure 6.

\section{Funding}

This work was supported by MIUR through PON-AIM Linea 1 (AIM1852570-1) and by Research Grant "Finanziamento giovanni ricercatori" 2020/2021 GNCS to I.M.B.

\section{Author's contributions}

IMB: Conceptualization, Methodology, Investigation, Writing-Original draft preparation. SA, IMB, RM, MAM and KP: Data Curation, Software, Validation, Writing-Reviewing and Editing. All authors discussed the results and contributed to the final manuscript.

\section{Acknowledgements}

This research has been accomplished within the UMI Group TAA "Approximation Theory and Application".

\section{References}

[1] https://www.who.int/health-topics/coronavirus.

[2] Bernoulli, D., Blower, S. An attempt at a new analysis of the mortality caused by smallpox and of the advantages of inoculation to prevent it. Reviews in medical virology, 14(5), 275-288, (2004). [CrossRef]

[3] Rahimi, I., Chen, F. and Gandomi, A.H. A review on COVID-19 forecasting models. Neural Computing and Applications, 1-11, (2021). [CrossRef]

[4] Akgül, A., Ahmed, N., Raza, A., et al. New applications related to Covid-19. Results in Physics, 20, 103663, (2021). [CrossRef]

[5] Farman, M., Akgül, A., Ahmad, A., Baleanu, D., Saleem, M.U. Dynamical Transmission of Coronavirus Model with Analysis and Simulation. CMES-Computer Modeling in Engineering and Sciences, 127(2), 753-769, (2021). [CrossRef]

[6] Amico, E., Bulai, I.M. How political choices shaped Covid connectivity: the Italian case study, Plos One, (2021). [CrossRef].

[7] Zeb, A., Alzahrani, E., Erturk, V.S., Zaman, G. Mathematical model for Coronavirus Disease 2019 (COVID-19) Containing Isolation Class. BioMed Research International, (2020). [CrossRef]

[8] Zhang, Z., Zeb, A., Hussain, S., Alzahrani, E. Dynamics of COVID-19 mathematical model with stochastic perturbation. Advances in Difference Equations, 2020(1), 1-12, (2020). [CrossRef] 
[9] Naik, P.A., Yavuz, M., Qureshi, S., Zu, J. \& Townley, S. Modeling and analysis of COVID-19 epidemics with treatment in fractional derivatives using real data from Pakistan. The European Physical Journal Plus, 135(10), 1-42, (2020). [CrossRef]

[10] Zhang, Z., Zeb, A., Egbelowo, O.F., Erturk, V.S. Dynamics of a fractional order mathematical model for COVID-19 epidemic. Advances in Difference Equations, 2020(1), 1-16, (2020). [CrossRef]

[11] Özköse, F., Yavuz, M. Investigation of interactions between COVID-19 and diabetes with hereditary traits using real data: A case study in Turkey. Computers in Biology and Medicine, 105044, (2021). [CrossRef]

[12] Nazir, G., Zeb, A., Shah, K., Saeed, T., Khan, R.A. \& Khan, S.I.U. Study of COVID-19 mathematical model of fractional order via modified Euler method. Alexandria Engineering Journal, 60(6), 5287-5296, (2021). [CrossRef]

[13] Bushnaq, S., Saeed, T., Torres, D.F.M., Zeb, A. Control of COVID-19 dynamics through a fractional-order model. Alexandria Engineering Journal, 60(4), 3587-3592, (2021). [CrossRef]

[14] Yavuz, M., Coşar, F.Ö, Günay, F., Özdemir, F.N. A new mathematical modeling of the COVID-19 pandemic including the vaccination campaign. Open Journal of Modelling and Simulation, 9(3), 299-321, (2021). [CrossRef]

[15] Zhang, Z., Zeb, A., Alzahrani, E. \& Iqbal, S. Crowding effects on the dynamics of COVID-19 mathematical model. Advances in Difference Equations, 2020(1), 1-13, (2020). [CrossRef]

[16] Li, X.P., Al Bayatti, H., Din, A. \& Zeb, A. A vigorous study of fractional order COVID-19 model via ABC derivatives. Results in Physics, 29, 104737, (2021). [CrossRef]

[17] Guan, J., Wei, Y., Zhao, Y., Chen, F. Modeling the transmission dynamics of COVID-19 epidemic: a systematic review. Journal of Biomedical Research, 34(6), 422-430, (2020). [CrossRef]

[18] Atangana, A., Araz, S.I. Mathematical model of COVID-19 spread in Turkey and South Africa: theory, methods, and applications. Advances in Difference Equations, 2020(1), 1-89, (2020). [CrossRef]

[19] Amaro, J.E., Dudouet, J., Orce, J.N. Global analysis of the COVID-19 pandemic using simple epidemiological models. Applied Mathematical Modelling, 90, 995-1008, (2021). [CrossRef]

[20] Buonomo, B., Della Marca, R. Effects of information-induced behavioural changes during the COVID-19 lockdowns: the case of Italy. Royal Society Open Science, 7(10), 201635, (2020). [CrossRef]

[21] Zhu, H., Li, Y., Jin, X., Huang, J., Liu, X., Qian, Y., Tan, J. Transmission dynamics and control methodology of COVID-19: A modeling study. Applied Mathematical Modelling, 89(2), 1983-1998, (2021). [CrossRef]

[22] Pellis, L. et al. \& University of Manchester COVID-19 Modelling Group. Challenges in control of Covid-19: short doubling time and long delay to effect of interventions. Philosophical Transactions of the Royal Society B, 376(1829), 20200264, (2021). [CrossRef]

[23] Sun, D., Duan, L., Xiong, J. \& Wang, D. Modeling and forecasting the spread tendency of the COVID-19 in China. Advances in Difference Equations, 2020(1), 1-16, (2020). [CrossRef]

[24] Giordano, G., Blanchini, F., Bruno, R., Colaneri, P., Di Filippo, A., Di Matteo, A. \& Colaneri, M. Modelling the COVID-19 epidemic and implementation of population-wide interventions in Italy. Nature medicine, 26(6), 855-860, (2020). [CrossRef]

[25] Pedersen, M.G., Meneghini, M. Data-driven estimation of change points reveals correlation between face mask use and accelerated curtailing of the first wave of the COVID-19 epidemic in Italy. Infectious Diseases, 53(4), 243-251, (2021). [CrossRef]

[26] Viguerie, A., et al. Simulating the spread of COVID-19 via a spatially-resolved susceptible-exposed-infected-recovered-deceased (SEIRD) model with heterogeneous diffusion. Applied Mathematics Letters, 111, 106617, (2021). [CrossRef]

[27] Ferguson, N., et al. Report 9: Impact of non-pharmaceutical interventions (NPIs) to reduce COVID19 mortality and healthcare demand.Imperial College London, (2020). [CrossRef]

[28] Chowdhury, R., Heng, K., Shawon, M.S.R., Goh, G., Okonofua, D., Ochoa-Rosales, C., ... \& Franco, O.H. Dynamic interventions to control COVID-19 pandemic: a multivariate prediction modelling study comparing 16 worldwide countries. European journal of epidemiology, 35(5), 389-399, (2020). [CrossRef]

[29] Jiao, J., Liu, Z., Cai, S. Dynamics of an SEIR model with infectivity in incubation period and homestead-isolation on the susceptible. Applied Mathematics Letters, 107, 106442, (2020). [CrossRef]

[30] Bai, Y., Yao, L., Wei, T., Tian, F., Jin, D.Y., Chen, L. \& Wang, M. Presumed Asymptomatic Carrier Transmission of COVID-19. JAMA, 323(14), 1406-1407, (2020). [CrossRef]

[31] Sakurai, A., Sasaki, T., Kato, S., Hayashi, M., Tsuzuki, S.I., Ishihara, T., et al. Natural History of Asymptomatic SARS-CoV-2 Infection. NEngl JMed, 383(9), 885-886, (2020). [CrossRef]

[32] Moore, S., Hill, E.M., Tildesley, M.J., Dyson, L., Keeling, M.J. Vaccination and non-pharmaceutical interventions for COVID-19: a mathematical modelling study. The Lancet Infectious Diseases, 21(6), 793-802, (2021). [CrossRef]

[33] Aniţa, S., Banerjee, M., Ghosh, S., Volpert, V. Vaccination in a two-group epidemic model. Applied Mathematics Letters, 119, 107197, (2021). [CrossRef]

[34] Estrada, E. COVID-19 and SARS-CoV-2: Modeling the present, looking at the future. Physics Reports, 869, 1-51, (2020). [CrossRef]

[35] Bulai, I.M. Modeling Covid-19 Considering Asymptomatic Cases and Avoided Contacts. Chapter in Trends in Biomathematics: Chaos and Control in Epidemics, Ecosystems, and Cells, Springer, (2021). [CrossRef]

[36] https://www.istat.it/storage/rapporto-annuale/2018/Rapportoannuale2018.pdf.

[37] https://raw.githubusercontent.com/pcm-dpc/COVID-19/master/dati-json/dpc-covid19-ita-andamento-nazionale.json

[38] Pedersen, M.G., Meneghini, M. Quantifying undetected COVID-19 cases and effects of containment measures in Italy, (2020).[Preprint].

[39] National Institute of Infectious Diseases Japan. Field Briefing: Diamond Princess COVID-19 Cases, 20 Feb Update. Last accessed March 12, 2020. Available from: https://www. niid.go.jp/niid/en/2019-ncov-e/9417-covid-dp-fe-02.html.

[40] O. Diekmann, J.A.P. Heesterbeek, J.A.J. Metz. On the definition and the computation of the basic reproduction ratio R 0 in models for infectious diseases in heterogeneous populations. Journal of mathematical biology, 28, 365-382, (1990). [CrossRef]

[41] P. Van den Driessche, J. Watmough. Reproduction numbers and sub-threshold endemic equilibria for compartmental models of disease transmission. Math Biosci, 180(1), 29-48, (2002). [CrossRef] 
Mathematical Modelling and Numerical Simulation with Applications (MMNSA) (http://www.mmnsa.org)

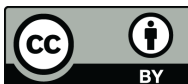

Copyright: (c) 2021 by the authors. This work is licensed under a Creative Commons Attribution 4.0 (CC BY) International License The authors retain ownership of the copyright for their article, but they allow anyone to download, reuse, reprint, modify, distribute, and/or copy articles in MMNSA, so long as the original authors and source are credited. To see the complete license contents, please visit (http://creativecommons.org/licenses/by/4.0/). 